PROCEEDINGS OF THE

AMERICAN MATHEMATICAL SOCIETY

Volume 128, Number 5, Pages 1371-1375

S 0002-9939(99)05326-5

Article electronically published on August 5, 1999

\title{
THE SPECTRAL PROPERTIES OF CERTAIN LINEAR OPERATORS AND THEIR EXTENSIONS
}

\author{
BRUCE A. BARNES
}

(Communicated by David R. Larson)

\begin{abstract}
Let $H$ be a Hilbert space with inner-product $(x, y)$, and let $R$ be a bounded positive operator on $H$ which determines an inner-product, $\langle x, y\rangle=$ $(R x, y), x, y \in H$. Denote by $H^{-}$the completion of $H$ with respect to the norm $\|x\|=\langle x, x\rangle^{1 / 2}$. In this paper, operators having certain relationships with $R$ are studied. In particular, if $T=S R^{1 / 2}$ where $S \in B(H)$, then $T$ has an extension $T^{-} \in B\left(H^{-}\right)$, and $T$ and $T^{-}$have essentially the same spectral and Fredholm properties.
\end{abstract}

\section{INTRODUCTION}

Throughout this paper, $H$ is a Hilbert space with inner-product $(x, y)$ and norm $\|x\|_{H}=(x, x)^{1 / 2}$. Assume that $\langle x, y\rangle$ is a bounded inner-product on $H$, so there exists $c>0$ such that for all $x, y \in H:|\langle x, y\rangle| \leq c\|x\|_{H}\|y\|_{H}$. Let $\|x\|=\langle x, x\rangle^{1 / 2}$, and let $H^{-}$be the completion of $H$ with respect to the norm $\|x\|$. Since the innerproduct $\langle x, y\rangle$ is bounded, it is well-known that there exists a positive operator $R \in B(H)$ such that

$$
\langle x, y\rangle=(R x, y) \quad \text { for all } x, y \in H .
$$

For future reference we note that

$$
\left\|R^{1 / 2} x\right\|_{H}=\|x\| \quad \text { for all } x \in H .
$$

Early work in this setting centered on operators of the form $T=S R$ where $S=S^{*} \in$ $B(H)$; see Chapters 15-17 of [Z]. For such $T,\langle T x, y\rangle=(R S R x, y)=(R x, S R y)=$ $\langle x, T y\rangle$ for all $x, y \in H$. An operator $T$ is symmetrizable with respect to an innerproduct $\langle x, y\rangle$, if $\langle T x, y\rangle=\langle x, T y\rangle$ for all $x, y \in H$. Thus the operator $T=S R$ above is symmetrizable.

The concept of a symmetrizable operator makes sense whenever there is a bounded inner-product on a Banach space. P. Lax studied symmetrizable operators in this more general setting in $\mathrm{L}$. He proved that when $T$ is symmetrizable, then $T$ has an extension $T^{-} \in B\left(H^{-}\right)$and $\sigma(T) \supseteq \sigma\left(T^{-}\right)$. Istratescu's book [I] Chapter 11] is a good source of information about symmetrizable operators and related ideas.

Here we restrict attention to the case where the underlying space is the Hilbert space $H$. Our main results concern operators of the form $T=S R^{1 / 2}$ where $S$ is

Received by the editors June 23, 1998.

1991 Mathematics Subject Classification. Primary 47A10, 47A30.

Key words and phrases. Bounded extension, spectrum, symmetrizable. 
an arbitrary operator in $B(H)$. It is shown that $T=S R^{1 / 2}$ has an extension to an operator $T^{-} \in B\left(H^{-}\right)$, and that $T$ and $T^{-}$have essentially the same basic operator properties (for example, they have the same spectrum).

\section{RESUlts}

We use the notation from the Introduction in what follows. In particular, $R$ is the positive operator determined by the bounded inner-product $\langle x, y\rangle$. We use the fact that $\mathbf{R}\left(R^{1 / 2}\right)$ is dense in $H$ (here, and in what follows, $\mathbf{R}(S)$ denotes the range of the operator $S$ ).

Theorem. (1)-(4) are equivalent for $T \in B(H)$ :

(1) $R T R^{-1}$ is bounded on $\mathbf{R}(R)$;

(2) there exists an operator $S \in B(H)$ such that $\langle T x, y\rangle=\langle x, S y\rangle$ for all $x, y \in H$;

(3) there exists an operator $S \in B(H)$ such that $R T=S R$;

(4) $T^{*}(\mathbf{R}(R)) \subseteq \mathbf{R}(R)$.

(5)-(8) are equivalent for $T \in B(H)$ :

(5) $R^{1 / 2} T R^{-1 / 2}$ is bounded on $\mathbf{R}\left(R^{1 / 2}\right)$;

(6) $T$ has an extension to a bounded operator $T^{-}$on $H^{-}$;

(7) there exists an operator $S \in B(H)$ such that $R^{1 / 2} T=S R^{1 / 2}$;

(8) $T^{*}\left(\mathbf{R}\left(R^{1 / 2}\right)\right) \subseteq \mathbf{R}\left(R^{1 / 2}\right)$. (9)-(12) are equivalent for $T \in B(H)$ :

(9) $T R^{-1 / 2}$ is bounded on $\mathbf{R}\left(R^{1 / 2}\right)$;

(10) $T$ has an extension to a bounded operator linear operator $T^{\sim}: H^{-} \rightarrow H$;

(11) there exists an operator $S \in B(H)$ such that $T=S R^{1 / 2}$;

(12) $T^{*}(H) \subseteq \mathbf{R}\left(R^{1 / 2}\right)$.

Proof. Clearly, $(3) \Rightarrow(1)$. Suppose that (1) holds. Let $S$ denote the bounded extension of $R T R^{-1}$ to all of $H$. It follows that $R T=S R$. Therefore (3) holds.

Assume that (2) holds. Then $(R T x, y)=(R x, S y)=\left(S^{*} R x, y\right)$ for all $x, y \in H$. Therefore, $R T=S^{*} R$, so (3) holds. Conversely, if $R T=S^{*} R$, then reversing the argument above, we have that (2) is true.

That $(3) \Rightarrow(4)$ is clear. Now assume that $T^{*}(\mathbf{R}(R)) \subseteq \mathbf{R}(R)$. Then $\mathbf{R}\left(T^{*} R\right) \subseteq$ $\mathbf{R}(R)$, so by the Douglas Range Inclusion Theorem [D], it follows that $T^{*} R=R S$ for some operator $S \in B(H)$. Taking adjoints, we have $R T=S^{*} R$, and thus (3) holds.

Assume that (5) holds. Then there exists $M>0$ such that

$$
\left\|R^{1 / 2} T R^{-1 / 2}\left(R^{1 / 2} x\right)\right\|_{H} \leq M\left\|R^{1 / 2} x\right\|_{H}
$$

for all $x \in H$. Thus by $\mathbb{*}^{*},\|T x\| \leq M\|x\|$ for all $x \in H$, and this implies (6). Also, this argument is reversible, so $(6) \Rightarrow(5)$.

Again, assume that (5) holds. Let $S$ be the bounded extension of $R^{1 / 2} T R^{-1 / 2}$ on $H$. It follows immediately that $S R^{1 / 2}=R^{1 / 2} T$. Thus (7) holds. Clearly (7) $\Rightarrow(5)$.

That $(7) \Rightarrow(8)$ is clear. Now assume that $T^{*}\left(\mathbf{R}\left(R^{1 / 2}\right)\right) \subseteq \mathbf{R}\left(R^{1 / 2}\right)$. Then $\mathbf{R}\left(T^{*} R^{1 / 2}\right) \subseteq \mathbf{R}\left(R^{1 / 2}\right)$. Again, applying the Range Inclusion Theorem, we have $T^{*} R^{1 / 2}=R^{1 / 2} S$ for some operator $S \in B(H)$. Taking adjoints in this equality we see that (7) is true.

Assume that (9) holds. Then there exists $M>0$ such that $\left\|T R^{-1 / 2}\left(R^{1 / 2} x\right)\right\|_{H} \leq$ $M\left\|R^{1 / 2} x\right\|_{H}$ for all $x \in H$. Thus by $\mathbb{*}$, $\|T x\|_{H} \leq M\|x\|$ for all $x \in H$, and this implies (10). Also, this argument is reversible, so $(10) \Rightarrow(9)$. 
Again, assume that (9) holds. Let $S$ be the bounded extension of $T R^{-1 / 2}$ on $H$. Then $T=S T^{1 / 2}$. Clearly $(11) \Rightarrow(9)$.

Finally, making use of the Range Inclusion Theorem as before, it is straightforward to check that $(11) \Leftrightarrow(12)$.

Corollary. Assume $T=S R^{1 / 2}$, where $S \in B(H)$.

(a) The operator $T$ has a bounded extension $T^{-} \in B\left(H^{-}\right)$with the property that $T^{-}\left(H^{-}\right) \subseteq H$.

(b) Let $E: H \rightarrow H^{-}$be the continuous embedding map, Ex=x for all $x$. Let $T^{\sim}: H^{-} \rightarrow H$ be as in part (10) of the Theorem. Then

$$
T^{\sim} \in B\left(H^{-}, H\right) ; \quad T=T^{\sim} E ; \quad T^{-}=E T^{\sim} .
$$

Proof. Since $R^{1 / 2} T=\left(R^{1 / 2} S\right) R^{1 / 2}$, the operator $T$ satisfies (7) in the Theorem. Then by $(7) \Rightarrow(6), T$ has a bounded extension $T^{-}$on $H^{-}$. Also by hypothesis, $T$ satisfies (11), so by (10), $T$ has a bounded extension $T^{\sim} \in B\left(H^{-}, H\right)$. Then clearly $T^{-}=E T^{\sim}$. It follows that $T^{-}\left(H^{-}\right) \subseteq H$. It is also clear that $T=T^{\sim} E$. This verifies both parts (a) and (b) of the Corollary.

For a bounded linear operator $S$, we use the notation:

$$
\begin{aligned}
\sigma(S) & =\text { the usual operator spectrum of } S \\
\sigma_{F}(S) & \equiv \text { the Fredholm spectrum of } S \\
& \equiv\{\lambda \in \mathbf{C}:(\lambda-S) \text { is not a Fredholm operator }\} ; \\
\sigma_{W}(S) & \equiv \text { the Weyl spectrum of } S \\
& \equiv\{\lambda \in \mathbf{C}:(\lambda-S) \text { is not a Fredholm operator of index zero }\} .
\end{aligned}
$$

In what follows, $R, T, T^{-}$, and $T^{\sim}$ are as in the Corollary.

Consequences. In I-III below, assume $T=S R^{1 / 2}$, where $S \in B(H)$.

I. By part (a) of the Corollary, $T^{-}\left(H^{-}\right) \subseteq H$. Applying [B1. Theorem 4(2)], we have:

(i) $\sigma(T)=\sigma\left(T^{-}\right)$;

(ii) $\sigma_{F}(T)=\sigma_{F}\left(T^{-}\right)$;

(iii) $\sigma_{W}(T)=\sigma_{W}\left(T^{-}\right)$.

Also: (iv) when $\lambda \neq 0, \mathbf{N}(\lambda-T)=\mathbf{N}\left(\lambda-T^{-}\right)$; here $\mathbf{N}(W)$ denotes the null space of the operator $W$.

II. By part (b) of the Corollary, $T=T^{\sim} E$ and $T^{-}=E T^{\sim}$. Therefore, $T$ and $T^{-}$have all the common operator properties described in [B3. In particular, when $\lambda \neq 0$ :

(i) $\lambda-T$ has a pseudoinverse $\Leftrightarrow \lambda-T^{-}$has a pseudoinverse [B3, Theorem $4]$

(ii) $\lambda-T$ has closed range $\Leftrightarrow \lambda-T^{-}$has closed range [B3, Theorem 5];

(iii) $\lambda$ is a pole of finite rank of the resolvent of $T \Leftrightarrow \lambda$ is a pole of finite rank of the resolvent of $T^{-}$[B3, Theorem 9].

III. Let $\mathcal{K}$ denote the set of all linear operators $J \in B(H)$ such that $J$ is compact, and $J$ has an extension $J^{-}$on $H^{-}$which is also compact. By I (iii), $\sigma_{W}(T)=$ $\sigma_{W}\left(T^{-}\right)$. It follows from [B2, Theorem 8] that

(i) $\sigma(T+J)=\sigma\left(T^{-}+J^{-}\right)$for all $J \in K$. 
IV. Now assume that $T=S R$ where $S=S^{*} \in B(H)$. Operators of this form are common in applications. As noted in the Introduction, $\langle T x, y\rangle=\langle x, T y\rangle$ for all $x, y \in H$. Since $T=\left(S R^{1 / 2}\right) R^{1 / 2}$, consequences I-III hold for $T$ and $T^{-}$, and in this case, $T^{-}$is selfadjoint.

Example 1. We give an example of a common situation in analysis where the results of this paper apply. Let $\mu$ be a measure defined on some $\sigma$-algebra of subsets of a set $\Omega$. Let $w$ be a weight function, $w \in L^{\infty}(\mu)$, with $w(x)>0 \mu$-a.e. Set $H=L^{2}(\mu)$. Consider the bounded inner-product on $H$ defined by

$$
\langle f, g\rangle \equiv \int_{\Omega} f g^{-} w d \mu \quad(f, g \in H)
$$

Let $R$ be the multiplication operator defined by: $R(f)=w f, f \in H$. Then $\langle f, g\rangle=(R f, g)$ for all $f, g \in H$.

Now let $K(x, t)$ be a kernel that determines a bounded integral operator $S$ on $L^{2}(\mu)$ :

$$
S(f)(x)=\int_{\Omega} K(x, t) f(t) d \mu(t), \quad f \in L^{2}(\mu) .
$$

Then consequences I-III apply to the operator $T=S R$,

$$
T(f)(x)=\int_{\Omega} K(x, t) w(t) f(t) d \mu(t), \quad f \in L^{2}(\mu) .
$$

When in addition $S=S^{*}$, then IV also applies to the operator $T$.

Example 2. There exist symmetrizable operators $T$ for which $\sigma(T)$ and $\sigma\left(T^{-}\right)$ can be very different. Now we modify an example due to J. Nieto in $[\mathbb{N}$ to verify this in our particular setting. Let $H$ be the weighted $l^{2}$-space of sequences $\left\{a_{k}\right\}_{k \geq 1}$ such that $\sum_{1}^{\infty} 4^{k}\left|a_{k}\right|^{2}<\infty$. The inner-product on $H$ is:

$$
\left(\left\{a_{k}\right\},\left\{b_{k}\right\}\right)=\sum_{1}^{\infty} 4^{k} a_{k} b_{k}^{-} .
$$

Consider the inner-product on $H$ defined by:

$$
\left\langle\left\{a_{k}\right\},\left\{b_{k}\right\}\right\rangle=\sum_{1}^{\infty} a_{k} b_{k}^{-} .
$$

It is easy to check that this inner-product is bounded on $H$, and that the positive operator $R$ such that $(R a, b)=\langle a, b\rangle$ is the multiplication operator $R\left(\left\{a_{k}\right\}\right)=$ $\left\{4^{-k} a_{k}\right\}$

Let $S$ and $B$ be the shift and backward shift on $H$, so $S\left(a_{1}, a_{2}, a_{3}, \ldots\right)=$ $\left(0, a_{1}, a_{2}, \ldots\right) ; B\left(a_{1}, a_{2}, a_{3}, \ldots\right)=\left(a_{2}, a_{3}, \ldots\right)$. Let $T=S+B$, and note that $T$ is selfadjoint relative to the inner-product $\langle a, b\rangle$. Let $H^{-}$be the completion of $H$ relative to the norm determined by the inner-product $\langle a, b\rangle$, so $H^{-}$is the usual sequence space $l^{2}$. Let $S^{-}, B^{-}$, and $T^{-}$denote the extensions of $S, B$, and $T$ to $l^{2}$. The extension $T^{-}$has real spectrum (in fact, $\sigma\left(T^{-}\right)=[-2,2]$ ). Now we compute the spectrum of $T$ in $B(H)$. Let $W: H \rightarrow l^{2}$ be defined by $W\left(\left\{a_{k}\right\}\right)=\left\{2^{k} a_{k}\right\}$. Note that $W$ is a linear isometry that maps $H$ onto $l^{2}$. A straightforward computation verifies that:

$$
W S W^{-1}=2 S^{-} ; \quad W B W^{-1}=\frac{1}{2} B^{-} ; \quad \text { so, } W T W^{-1}=2 S^{-}+\frac{1}{2} B^{-} .
$$


These operators act on $l^{2}$. The spectrums of these operators have been computed; see [N] Prop. 2]. Using this result, we have that $\sigma\left(W T W^{-1}\right)=\Gamma \equiv$ $\left\{\right.$ all the numbers in the complex plane which are inside or on the ellipse $\frac{4}{25} x^{2}+\frac{4}{9} y^{2}$ $=1\}$. Thus,

$$
\sigma(T)=\Gamma \supseteq[-2,2]=\sigma\left(T^{-}\right) .
$$

We note that in contrast, the weighted shift and weighted backward shift, $S R^{1 / 2}$ and $B R^{1 / 2}$, have the same spectral properties on $H$ and $H^{-}$.

Example 3. Let $H, H^{-}$, and $R$ be as in Example 2 We construct an example of an operator $W \in B(H)$ such that $T=W R^{1 / 2}$ does not have an adjoint in $B(H)$ with respect to the inner-product $\langle a, b\rangle$. Not only does $T$ have a bounded extension $T^{-}$on $H^{-}$[Corollary], but also $T$ and $T^{-}$satisfy the consequences I, II, and III.

Let $e_{k}$ denote the vector in $H$ with $k$ th coordinate 1 and with all other coordinates 0 . Note that $\left\|e_{k}\right\|_{H}=2^{k}$ for all $k$, and that the sequence $\left\{2^{-k} e_{k}\right\}_{k \geq 1}$ is an orthonormal basis for $H$. Define $W$ on this sequence by $W\left(e_{k}\right)=0$ if $k \neq m^{2}$ for $m \geq 1$, and $W\left(2^{-m^{2}} e_{m^{2}}\right)=2^{-m} e_{m}$, otherwise. Clearly $W \in B(H)$. Now we show that $R W R^{1 / 2} R^{-1}$ is not bounded on $\mathbf{R}(R)$, so part (1) of the Theorem cannot hold for $T$. Since $R^{-1 / 2}\left(e_{k}\right)=2^{k} e_{k}$,

$$
R W R^{-1 / 2}\left(2^{-m^{2}} e_{m^{2}}\right)=R W\left(e_{m^{2}}\right)=R\left(2^{\left(m^{2}-m\right)} e_{m}\right)=4^{-m} 2^{\left(m^{2}-m\right)} e_{m} .
$$

Finally, $\left\|4^{-m} 2^{\left(m^{2}-m\right)} e_{m}\right\|_{H}=4^{-m} 2^{m^{2}} \rightarrow \infty$ as $m \rightarrow \infty$. This proves that $T=$ $W R^{1 / 2}$ does not have an adjoint in $B(H)$ with respect to the inner-product $\langle a, b\rangle$.

\section{REFERENCES}

[B1] B. Barnes, The spectral and Fredholm theory of extensions of bounded linear operators, Proc. Amer. Math. Soc. 105 (1989), 941-949. MR 89i:47008

[B2] B. Barnes, Essential spectra in a Banach algebra applied to linear operators, Proc. of the Royal Irish Acad. 90A (1990), 73-82. MR 93b:46087

[B3] B. Barnes, Common operator properties of the linear operators $R S$ and SR, Proc. Amer. Math. Soc. 126 (1998), 1055-1061. MR 98f:47003

[D] R. Douglas, On majorization, factorization, and range inclusion of operators on Hilbert space, Proc. Amer. Math. Soc. 17 (1966), 413-415. MR 34:3315

[I] V. Istratescu, Introduction to Linear Operator Theory, Marcel-Dekker, New York, 1981. MR 83d:47002

[L] P. Lax, Symmetrizable linear transformations, Comm. Pure and Applied Math. 7 (1954), 633-647. MR 16:823d

[N] J. Nieto, On the essential spectrum of symmetrizable operators, Math. Annalen 178 (1968), 145-153. MR 38:1544

[Z] A. C. Zaanen, Linear Operators, North-Holland Pub. Co., Amsterdam, 1953.

Department of Mathematics, University of Oregon, Eugene, Oregon 97403

E-mail address: barnes@math.uoregon.edu 